\title{
ORIGINAL RESEARCH \\ Clinical Characteristics and MR Imaging Features of Nonalcoholic Wernicke Encephalopathy
}

\author{
G.-q. Fei \\ C. Zhong \\ L. Jin \\ J. Wang \\ Yuhao Zhang \\ X. Zheng \\ Yuwen Zhang \\ Z. Hong
}

BACKGROUND AND PURPOSE: Nonalcoholic Wernicke encephalopathy (WE) is prone to be underestimated in clinical practice. The purpose of this study was to improve its awareness and early accurate diagnosis.

MATERIALS AND METHODS: We conducted a retrospective review of the cases of 12 patients with nonalcoholic WE, consisting of clinical characteristics and MR imaging features as well as follow-up after administration of thiamine.

RESULTS: Patients with mild coma or lethargy (7/12) exhibited typical MR features of symmetric brain paraventricular damage. Patients without disturbances of consciousness or who only had drowsiness (3/12) exhibited a lesion of the periaqueductal area only. In addition to typical MR manifestations, symmetric cortical involvement was observed in 2 of 12 patients with deep coma. Gadolinium enhancement of the mammillary bodies was observed in 2 of 3 patients. No atrophy of the mammillary bodies and cerebellar vermis was found in any patients. Of 10 patients without deep coma and cortical damage, 2 missed the follow-up and 8, who recovered clinically, also showed accordant resolution of abnormal hyperintense signal intensity on T2-weighted and fluid-attenuated inversion recovery images within 2 weeks to 1 year after thiamine supplementation. Two patients with deep coma and cortical damage showed a poor prognosis: 1 patient died 15 days after being diagnosed with WE, and the other entered a persistent vegetative state during a follow-up of 2 years.

CONCLUSION: Typical symmetric damage of the mammillary bodies and brain paraventricular regions may permit a specific diagnosis of nonalcoholic WE. In all patients, no atrophy of the mammillary bodies and cerebellar vermis was found. Cortical involvement in patients with nonalcoholic WE may be indicative of irreversible lesions and a poor prognosis.
$\mathbf{W}$ ernicke encephalopathy (WE) is a serious but curable neurologic disease caused by thiamine deficiency that occurs most commonly in people with alcoholism. In addition to the conditions with basic diseases and complications, the prognosis of patients with WE depends on the commencing time of thiamine supplementation, the most important factor relative to a curative effect. Therefore, early accurate diagnosis on WE is critical in clinical practice. In general, it is straightforward that we diagnose typical WE according to the background on alcoholism and a triad of neuro-ophthalmologic manifestations, ataxia, and global confusion. However, in clinical practice, the typical course of WE is limited and constitutes less than $16 \% .^{1-2}$ Some cases of WE become irreversible because they are often delayed in diagnosis or are misdiagnosed based on ambiguous symptoms such as dizziness, weakness, indifference, anorexia, and disturbance in memory. The diagnosis of nonalcoholic WE is usually more difficult than that of alcoholic WE. Some studies reported that only $20 \%$ or less of patients with nonalcoholic WE were definitely diagnosed premortem. ${ }^{3}$ It shows that nonalcoholic WE requires more awareness and heightened clinical suspicion. In addition, most clinical data about WE are from the studies of alcoholic WE, and nonalcoholic WE has been reported only in isolated cases. ${ }^{4-6}$ Herein, we report the cases of 12 patients with

Received March 26, 2007; accepted after revision May 7.

From the Department of Neurology (G.-q.F., C.Z., L.J., Yuhao Zhang, X.Z., Yuwen Zhang), Zhongshan Hospital and Shanghai Medical College, Fudan University, Shanghai, China; and Department of Neurology (J.W., Z.H.), Huashan Hospital and Shanghai Medical College, Fudan University, Shanghai, China.

Please address correspondence to Chunjiu Zhong, Department of Neurology, Zhongshan Hospital and Shanghai Medical College, Fudan University, Shanghai, 200032, China; e-mail: zhongcj@163.com

DOI 10.3174/ajnr.A0827 nonalcoholic WE diagnosed in our hospital from 1999 to 2006. The aim of our study was to improve awareness and early accurate diagnosis of nonalcoholic WE.

\section{Clinical Data}

\section{General Clinical Materials}

We enrolled 12 patients ( 8 male and 4 female) with WE, with an average age of 43.9 years (range, $16-69$ years). All patients denied a history of alcoholic abuse and overeating binges before the onset of WE. One patient who had a continual headache, vertigo, nausea, vomiting, and less intake of food for 42 days was diagnosed with a cavernoma in the pons on cranial CT (Fig. 2E) and was injected intravenously with glucose in saline discontinuously before the onset of WE. The others had a history of therapeutic fasting before WE as a result of acute pancreatitis ( 9 patients), gastroduodenectomy of a duodenal papillar tumor ( 1 patient), or enterolysis ( 1 patient). The time of therapeutic fasting ranged from 16 to 34 days, and the average time was $28.18 \pm 8.18$ days $(x \pm S D)$. During the fasting, the patients received total parenteral nutrition without thiamine supplementation.

\section{Neurologic Manifestations}

Initial central nervous system (CNS) symptoms appeared generally within 14 to 34 days after fasting in 11 patients with therapeutic fasting and 42 days after taking less food and being injected intravenously with glucose in saline discontinuously in 1 patient. Initial CNS symptoms included dizziness ( 5 patients), drowsiness ( 2 patients), apathy (3 patients), disturbance in memory (3 patients), unsteadiness with walking ( 3 patients), psychotic symptoms (2 patients), diplopia (1 patient), and decreased visual acuity (1 patient). When acute WE was suspected, 10 patients had disturbances of consciousness, including 1 


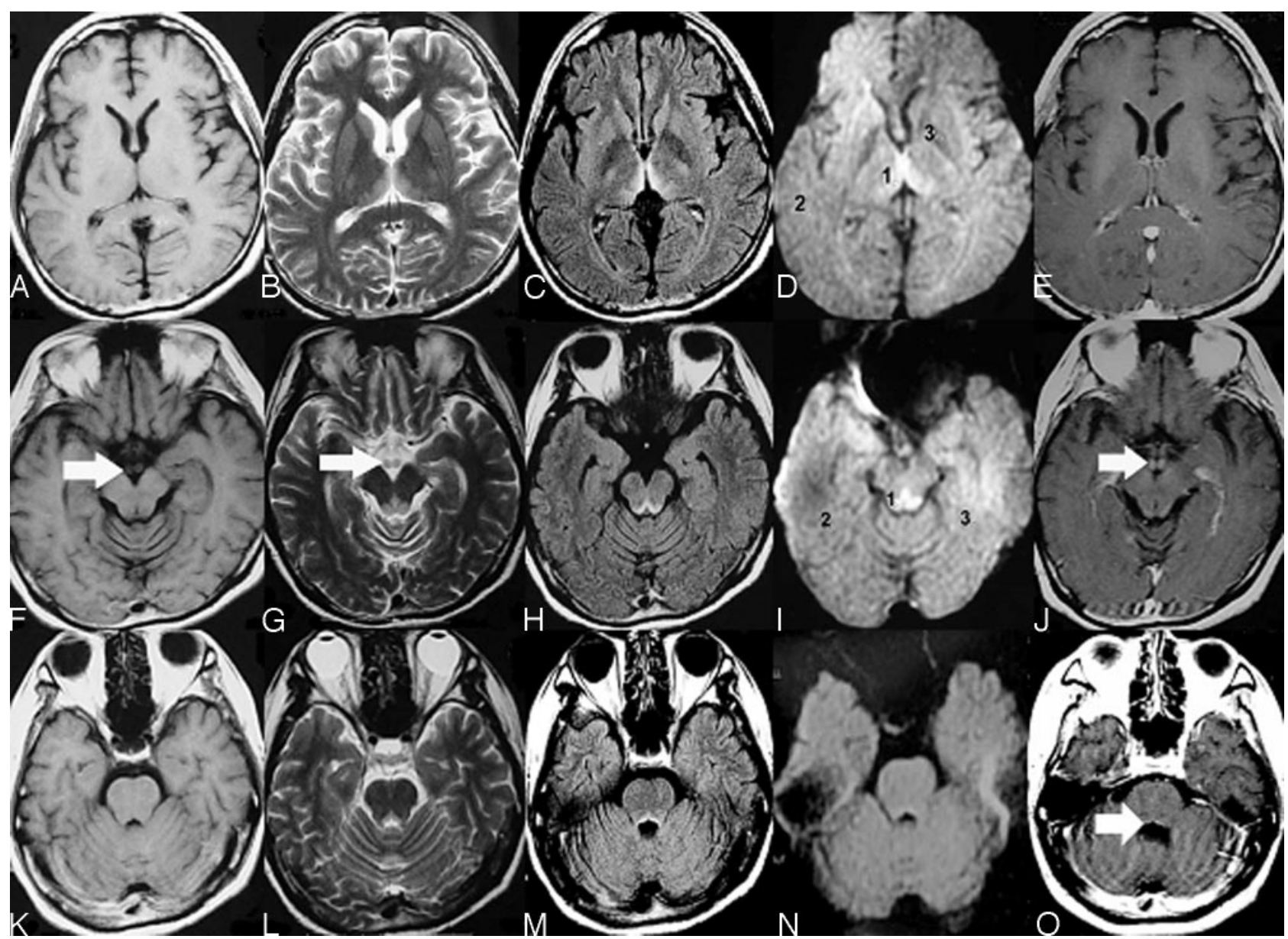

Fig 1. Typical MR imaging features of nonalcoholic Wernicke encephalopathy. Axial T1-weighted images $(A, F, K)$ show that no abnormal signal intensity was found. Axial T2-weighted $(B, G, L)$, and FLAIR $(C, H, M)$ images show increased signal intensity symmetric within the medial thalami $(B, C)$, periaqueductal area $(G, H)$, and floor of the fourth ventricle $(L, M)$. DW $(D, I, M)$ imaging shows only slightly increased signal intensities within the bilateral thalami and periacqueductal area but no abnormal signal intensity in the other brain regions. (The $A D C$ values of sites 1,2 , and 3 in the $D$ image were $620.19 \pm 27.39,513.29 \pm 9.60$, and $381.92 \pm 25.73$, respectively; the ADC values of sites 1,2 , and 3 in the / image were $657.00 \pm$ $43.13,442.23 \pm 15.43$, and $494.94 \pm 12.66$, respectively.) Contrasting images $(E, J, O)$ show that enhancement of the mammillary bodies and at the floor of the fourth ventricle by gadolinium contrast medium is found $(J)$. No atrophy of the mammillary bodies $(F, G, I, J)$ and cerebellar vermis $(K, L, M, N, O)$ was found.

with drowsiness, 3 with lethargy, 4 with mild coma, and 2 with deep coma. In addition to disturbances of consciousness, some patients had other symptoms: 1 with decreased visual acuity binocularly, 1 with diplopia, 2 with psychotic symptoms, 3 with ataxia, 3 with disturbances in memory, 4 with abnormal ocular movement, and 7 with nystagmus.

Of all patients, only 2 had a typical triad (neuro-ophthalmologic manifestation, ataxia, and global confusion) and 3 had disturbances of consciousness only, without focal neurologic signs. In accordance with clinical characteristics and the data of auxiliary investigations, metabolic disorders such as hypoglycemic shock, hepatic encephalopathy, and pulmonary encephalopathy were excluded.

\section{MR Imaging Examination and Findings}

We immediately performed cranial MR imaging when acute WE was suspected, 3 to 14 days after the occurrence of initial CNS symptoms. MR imaging with a $1.5 \mathrm{~T}$ MR scanner (GE Healthcare, Milwaukee, Wis) consisted of T1-weighted (TR, 500; TE, 9), T2-weighted (TR, 4000; effective TE, 99), fluid-attenuated inversion recovery (FLAIR) (TR, 10002; effective TE, 148), and diffusion-weighted MR imaging (DWI; TR, 2900; effective TE, 84). All patients were examined with T1- and T2-weighted imaging, 10 with FLAIR imaging, 4 with DWI imaging, and 3 patients ( 1 with drowsiness, 2 with mild coma) with enhanced scanning by gadolinium contrast.

All 7 patients with mild coma or lethargy exhibited typical MR imaging of acute Wernicke encephalopathy that showed areas of increased T2-weighted or FLAIR signals, or both, symmetrically surrounding the aqueduct and third ventricle, floor of the fourth ventricle, and medial thalami (Fig. 1). Only increased signal intensities on T2-weighted or FLAIR images, or both, surrounding the aqueduct were observed in 3 patients without disturbances of consciousness or who only had drowsiness (Fig. 2). Two patients with deep coma exhibited increased T2-weighted and FLAIR signal intensities, not only in the bilateral medial thalami but also in the motor cortex (Fig 3). Four patients ( 2 with mild coma and 2 with deep coma) had accompanied abnormal signal intensity in the capita of the caudate nuclei. No atrophy of the cerebellar vermis and mammillary bodies was observed in any patient (Fig. 1).

Among the 4 patients who underwent DWI imaging, 3 with mild coma exhibited only slightly increased signal intensities within the bilateral thalami and periaqueductal region. No abnormal signal intensity was found in other brain regions and in the other patient who had no disturbances of consciousness, which showed that DWI imaging may be insensitive to WE lesions (Figs $1 D, 1 I$, and $1 N$; see 


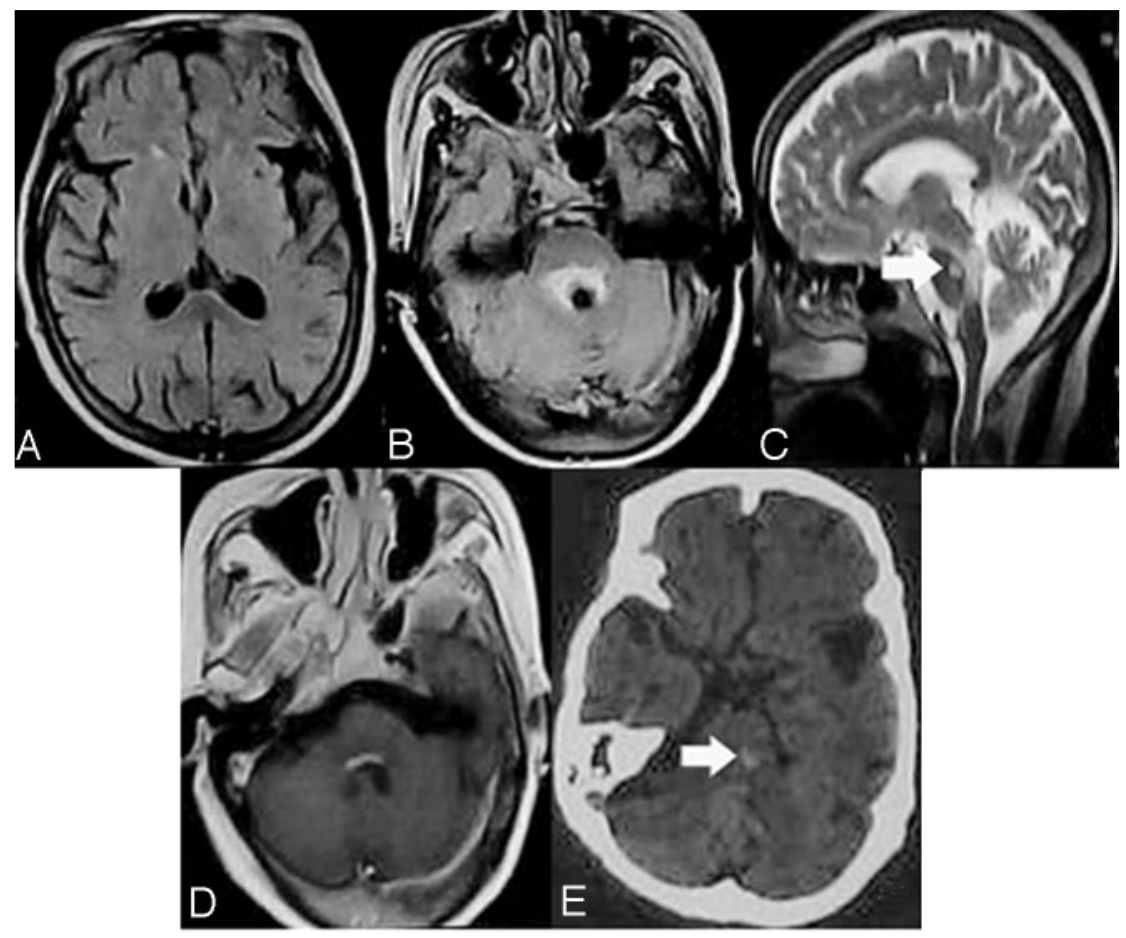

Fig 2. MR imaging manifestations in patients with drowsiness or without disturbances of consciousness. FLAIR images show that only regional damage surrounding the aqueduct ( $B$, $C)$ and the floor of fourth ventricle $(D$, enhanced by gadolinium contrast) is observed, and no abnormal signal intensity was found in the bilateral medial thalami (A). Arrowheads indicate cavernous hemangioma in the pons $(C, E$; $E$ was a CT)

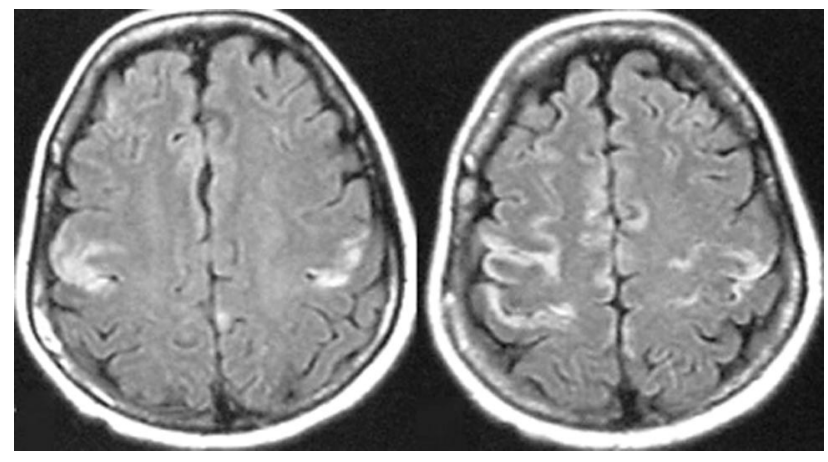

Fig 3. MR imaging images demonstrating cortical damage in patients with deep coma.

apparent diffusion coefficients [ADCs] in the figure legend). Among the 3 patients who underwent enhanced scanning with gadolinium contrast, 2 with mild coma were observed to have enhancing signal intensity in the mammillary bodies, which indicated acute disruption of the blood-brain barrier during acute WE, and nonenhancing signal intensity of the other brain zones (Figs $1 E, 1 \mathrm{~J}$, and 1O). One patient with mild coma (Fig 1O) and the other patient with drowsiness exhibited slight enhancing signal intensity at the floor of the fourth ventricle (Fig. 2).

\section{Treatment and Follow-up}

When WE was suspected in our patients, we immediately administered 100 to $200 \mathrm{mg}$ of intravenous or intramuscular thiamine and, thereafter, $100 \mathrm{mg}$ intravenous or intramuscular thiamine per day for 5 to 15 consecutive days. All patients were followed for 1 to 2 years until they recovered or their pathologic condition was stable, except for 1 patient who died and 2 patients who were missing. During follow-up, we administered fursultiamine (150 mg per day) via the gastrointestinal tract and conducted neurologic and MR examinations once every 3 months.

Follow-up showed 8 patients without deep coma and cortical damage recovered fully, both in the clinical setting and on cranial MR imaging within a 12-month duration (Fig. 4). It is noteworthy that the follow-up MR images of all 8 patients did not exhibit atrophy of the mammillary bodies and cerebellar vermis. The 2 patients with deep coma and cortical damage had a poor prognosis. One patient died 15 days after initial neurologic manifestations, and the other, whose MR imaging follow-up exhibited progressive atrophy of the whole brain (Fig. 5), entered a permanent vegetative state. These findings show that deep coma and cortical damage might be indicative of irreversible damage and a poor prognosis.

\section{Discussion}

Our study indicated that we should pay more attention to some predisposing factors for the development of nonalcoholic WE, such as prolonged therapeutic fasting, parenteral nutrition, and intravenous infusion of glucose. A high index of suspicion for nonalcoholic WE is required in patients with these factors. Apart from these factors, the literature reports that nonalcoholic WE could occur after most gastrointestinal surgical procedures, including gastric bypass surgery, ${ }^{7-9}$ gastrojejunostomy, ${ }^{10}$ gastrectomy, ${ }^{11}$ colectomy, ${ }^{12}$ and even therapy with an intragastric balloon. ${ }^{13}$ In addition, hyperemesis gravidarum, ${ }^{14-16}$ chemical therapy, ${ }^{17}$ anorexia nervosa, ${ }^{18}$ AIDS, ${ }^{19}$ and even wrong formula feeding ${ }^{20}$ were also reported as predisposing factors (Table 1). ${ }^{6,21-27}$ Because the healthy body's reserves of thiamine are sufficient for up to 18 days, any condition that induces thiamine deficiency lasting 2 to 3 weeks may lead to WE. Prophylactic use of thiamine with nutritional guidance should be recommended to avoid the development of WE in patients with possible thiamine deficiency. In this aspect, Mayo Clinic offered us a valuable experience in its series of bariatric surgery: no patients experienced WE. ${ }^{28}$

In clinical practice, WE is still underestimated because of either a relatively nonspecific clinical presentation of the disease in some cases or poorly recognized clinical symptoms and signs. ${ }^{29}$ Compared with alcoholic WE, patients with nonalco- 


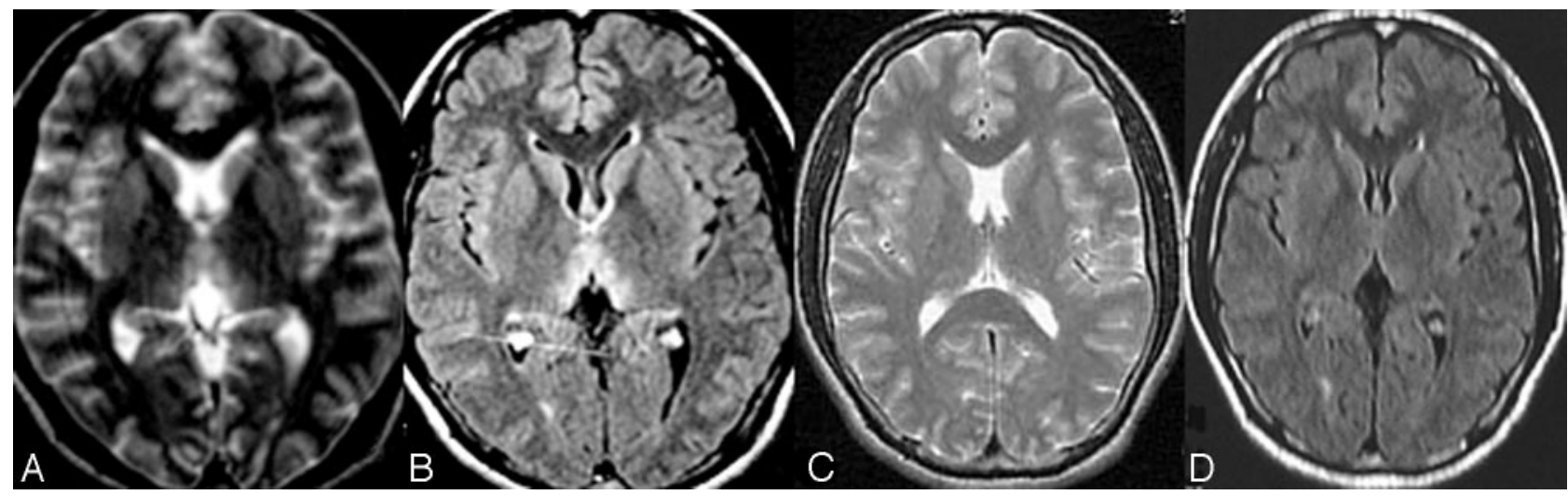

Fig 4. MR imaging follow-up of a patient with mild coma. Axial images show symmetric T2-weighted and FLAIR increased signal intensity in the medial thalami in the acute phase of WE $(A, B)$ and normal signal intensities 1 year after thiamine supplementation $(C, D)$.

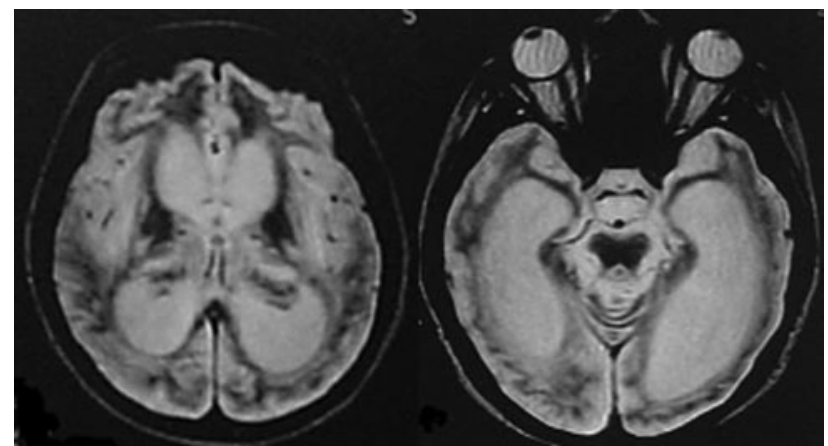

Fig 5. MR imaging follow-up of a patient with deep coma. The images show progressive atrophy 2 years after the onset of WE.

holic WE may be prone to a misdiagnosis or a delay in diagnosis because they manifest a mostly atypical clinical process. $^{2-6,30}$ Autopsy studies have showed that approximately $75 \%$ to $80 \%$ of cases in adults and $58 \%$ of cases in children have been missed by routine clinical examination. ${ }^{31}$ Our study showed a consistent result with the literature ${ }^{2-5}$ that most patients with nonalcoholic WE exhibited nonspecific clinical manifestations in the early stages of the disease. Only 2 of our 12 patients manifested a typical triad, and even 3 of the 12 patients exhibited disturbances of consciousness without signs of focal CNS damage in the whole course of the disease. Table 2 lists the complicated clinical manifestations of nonalcoholic WE according to our experience and reports in the literature.

The rate of early accurate diagnosis of nonalcoholic WE remains low and needs to be improved. ${ }^{32}$ Although our study attained a high rate of early accurate diagnosis of $83 \%, 2$ of the first 3 patients were still delayed to diagnose and their condition became irreversible: 1 patient died, and the other entered a persistent vegetative state with progressive atrophy of the whole brain during 2 years of follow-up (Fig 5). We believe that more awareness and heightened clinical suspicion of nonalcoholic WE must be required and the possibility of WE must be considered if a patient who has a potential status of thiamine deficiency exhibits dizziness, weakness, indifference, anorexia, memory disturbance, and so on. Because it is very safe and economical, tentative therapy of enough intramuscular or intravenous thiamine (100 mg or more daily) should be proposed to prevent the aggravation of thiamine deficiency when the situation is ambiguous.
From a theoretical standpoint, the determination of blood transketolase activity and thiamine phosphate or pyrophosphate can reflect the status of thiamine existing in the human body and be an accurate index in the diagnosis of WE. ${ }^{33}$ However, only a few hospitals have developed methods of determination as diagnosing tools because of technological complexity and cost. So far, WE is still diagnosed according to the history of the disease and therapeutic effect of thiamine supplementation. ${ }^{7}$ In 1998, Antunez et $\mathrm{al}^{34}$ reported that MR imaging exhibiting increased T2 signal intensity in the periaqueductal regions of the midbrain and paraventricular regions of the thalamus had a sensitivity of $53 \%$ and a specificity of $93 \%$ for the diagnosis of acute WE. Recently, some case studies ${ }^{6,35-38}$ reported the analogous result that typical MR imaging showed areas of increased T2-weighted and FLAIR signals symmetrically surrounding the aqueduct and the third ventricle, at the floor of fourth ventricle, in the medial thalami, which was consistent with the localization of pathologic lesions. ${ }^{38}$ Our study showed a consistent result with the literature. In addition, we found that 2 of 3 of our patients injected with gadolinium contrast medium were found to have enhanced signal intensity of the mammillary bodies. This finding is consistent with that in the previous literature ${ }^{30,39-40}$ that enhancement of mammillary bodies by contrast medium might be helpful to diagnose acute WE. However, it differs from the previous reports ${ }^{3,41-42}$ about alcoholic WE because the atrophy of the mammillary bodies and cerebellar vermis was not found during the acute stage and at follow-up of all patients in this study. The reasons leading to this difference have been explained in our previous report and may be the following: ${ }^{6,38,43}$ The cerebellar vermis and mammillary bodies may be susceptible to thiamine deficiency in patients with alcoholism ${ }^{2}$; these patients may have had previous attacks, and MR imaging findings in the acute phase of WE are contaminated by previous injury. MR imaging studies among nonalcoholic patients likely represent the evolution of pathologic findings in the first onset of WE because of the absence of previous attacks.

A few studies have reported atypical MR imaging presentations of nonalcoholic WE that exhibited lesions of the bilateral red nuclei and cerebellar dentate nuclei, ${ }^{44}$ putamen nuclei, ${ }^{17}$ capita of caudate nuclei, ${ }^{17,45}$ and cortex, ${ }^{8,17,30,46}$ in addition to typical injuries of the periaqueductal area and bi- 


\begin{tabular}{|c|c|}
\hline Cause & Author, Year, and Reference \\
\hline Bariatric surgery & Singh S, 20077; Nolli M, 2005\%; Loh Y, $2004^{9}$ \\
\hline Gastrectomy and gastrojejunostomy & Worden RW, 2006 ${ }^{10}$; Karapanayiotides T, 2006 ${ }^{11}$ \\
\hline Therapy with intragastric balloon & Chaves LC, $2002^{13}$ \\
\hline Colectomy & Pagnan L, $1998^{12}$ \\
\hline Hyperemesis gravidarum & Chiossi G, 2006 ${ }^{14}$; Selitsky T, 2006 ${ }^{15}$; Spruill SC, $2002^{16}$ \\
\hline Parenteral nutrition, hyperalimentation, prolonged intravenous glucose infusion & Zhong C, 20056; Attard 0, 2006²1; Francini-Pesenti F, $2007^{22}$ \\
\hline Prolonged starvation & Basoğlu M, 2006²3; Drenick EJ, $1966^{24}$ \\
\hline Anorexia nervosa & Peters TE, $2007^{18}$ \\
\hline Terminal tumor & Yae S, $2005^{25}$ \\
\hline AIDS & Butterworth RF, $1991^{19}$ \\
\hline Hemodialysis & Ihara M, $1999^{26}$ \\
\hline Chemical therapy & D'Aprile P, $2000^{17}$ \\
\hline Allogenic stem cell transplantation & Baek JH, $2005^{27}$ \\
\hline Wrong formula feeding & Fattal-Valevski A, $2005^{20}$ \\
\hline Brain stem disease & Fei G, 2007 \\
\hline
\end{tabular}

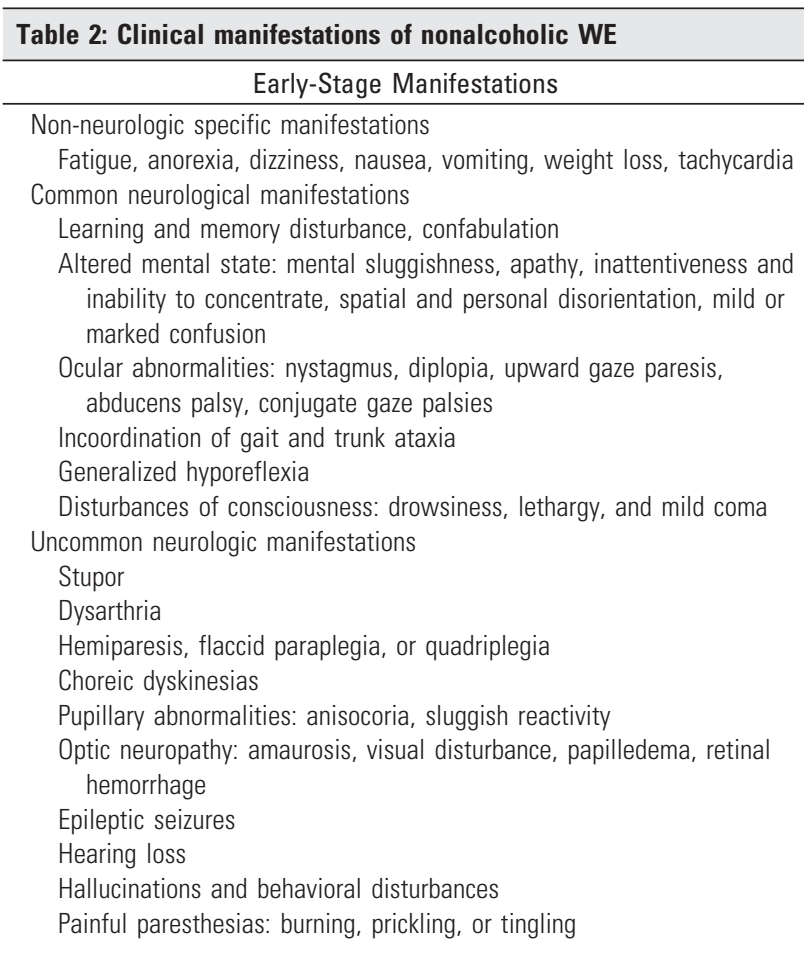

Late-Stage Manifestations

\section{Hypotension}

Hyperthermia or hypothermia

Deep coma

lateral medial thalami. We have not found any lesions of the red nuclei, putamen nuclei, and cerebellar dentate nuclei in this series. However, when combining the review of the literature with our data, we believe that cortical involvement may be indicative of irreversible damage because most patients with nonalcoholic WE who had coexistent cortical damage encountered a poor prognosis, ${ }^{6,8,30}$ though cases reported by Doss $^{46}$ and D'Aprile ${ }^{17}$ indicated the opposite result. A possible explanation for this divergence has been discussed in our previous report. ${ }^{6}$

Apart from WE, the diseases with symmetric lesions of the bilateral medial thalami also include bilateral paramedian thalamic syndrome, ${ }^{47}$ top-of-the-basilar syndrome (TOBS), ${ }^{48}$ cerebral venous thrombosis, ${ }^{49}$ viral encephalitis, ${ }^{50}$ acute dis- seminated encephalomyelitis (ADEM), ${ }^{30,51}$ and atypical Creutzfeldt- Jakob disease. ${ }^{52}$ All these diseases can be differentiated from WE through clinical characteristics and MR imaging. Patients with TOBS usually have vascular risk factors and lesions of the midbrain, upper pons, and bilateral occipital and temporal lobes. Inflammatory encephalopathy such as viral encephalitis or ADEM coexists with fever, multifocal demyelinating lesions of the brain and spinal cord, and increased cell numbers in the CSF. It is easy to differentiate viral encephalitis and ADEM from WE by symmetric involvement of the periventricular sites, which may permit a specific diagnosis of WE that includes the bilateral medial thalami, the periaqueductal gray matter, the floor of the fourth ventricle, and the mammillary bodies. ${ }^{35}$ Bilateral paramedial thalamic syndrome can include several diseases such as bilateral paramedial thalamic infarctions ${ }^{53}$ and reversible bilateral thalamic lesions $^{54}$ that possess a typical course of acute stroke and may result from systemic hypotension or hypoxia. Atypical Creutzfeldt-Jakob disease is a rare disorder with a progressive and irreversible course.

In summary, nonalcoholic WE exhibiting mostly complicated clinical manifestations and an atypical progression of the disease is prone to be underestimated. Symmetric damage of the mammillary bodies (with hyperintense signal intensity or gadolinium enhancement, or both) and brain paraventricular regions (including the medial thalami, floor of the fourth ventricle, and periaqueductal areas) are typical MR imaging manifestations of nonalcoholic WE that may permit a specific diagnosis. Nonalcoholic WE differs from alcoholic WE because no atrophy of the mammillary bodies and cerebellar vermis is found. In addition, cranial MR imaging is of great value in the judgment of the prognosis of this disorder. Coexistent cortical damage in patients with nonalcoholic WE may be indicative of irreversible lesions and a poor prognosis.

\section{References}

1. Harpe CG, Giles M, Finlay-Jones R. Clinical signs in the Wernicke-Korsakoff complex: a retrospective analysis of 131 cases diagnosed at necropsy. J Neurol Neurosurg Psychiatry 1986;49:341-45

2. Sun GH, Yang YS, Liu QS, et al. Pancreatic encephalopathy and Wernicke encephalopathy in association with acute pancreatitis: a clinical study. World J Gastroenterol 2006;12:4224-27

3. Gui QP, Zhao WQ, Wang LN. Wernicke's encephalopathy in nonalcoholic patients: clinical and pathologic features of three cases and literature reviewed. Neuropathology 2006;26:231-35 
4. Ogershok PR, Rahman A, Nestor S, et al. Wernicke encephalopathy in nonalcoholic patients. Am J Med Sci 2002;323:107-11

5. Munir A, Hussain SA, Sondhi D, et al. Wernicke's encephalopathy in a nonalcoholic man: case report and brief review. Mt Sinai J Med 2001;68:216-18

6. Zhong C, Jin L, Fei G. MR imaging of nonalcoholic Wernicke encephalopathy: a follow-up study. AJNR Am J Neuroradiol 2005;26:2301-05

7. Singh S, Kumar A. Wernicke encephalopathy after obesity surgery: a systematic review. Neurology 2007;68:807-11

8. Nolli M, Barbieri A, Pinna C, et al. Wernicke's encephalopathy in a malnourished surgical patient: clinical features and magnetic resonance imaging. Acta Anaesthesiol Scand 2005;49:1566-70

9. Loh Y, Watson WD, Verma A, et al. Acute Wernicke's encephalopathy following bariatric surgery: clinical course and MRI correlation. Obes Surg 2004;14:129-32

10. Worden RW, Allen HM. Wernicke's encephalopathy after gastric bypass that masqueraded as acute psychosis: a case report. Curr Surg 2006;63:114-16

11. Karapanayiotides T, Zaloni I, Thomaides T. Delayed development of Wernicke encephalopathy after gastrectomy. Arch Neurol 2006;63:1026-27

12. Pagnan L, Berlot G, Pozzi-Mucelli RS. Magnetic resonance imaging in a case of Wernicke's encephalopathy. Eur Radiol 1998;8:977-80

13. Chaves LC, Faintuch J, Kahwage S, et al. A cluster of polyneuropathy and Wernicke-Korsakoff syndrome in a bariatric unit. Obes Surg 2002;12:328-34

14. Chiossi G, Neri I, Cavazzuti M, et al. Hyperemesis gravidarum complicated by Wernicke encephalopathy: background, case report, and review of the literature. Obstet Gynecol Surv 2006;61:255-68

15. Selitsky T, Chandra P, Schiavello HJ. Wernicke's encephalopathy with hyperemesis and ketoacidosis. Obstet Gynecol 2006;107:486-90

16. Spruill SC, Kuller JA. Hyperemesis gravidarum complicated by Wernicke's encephalopathy. Obstet Gynecol 2002;99:875-77

17. D'Aprile P, Tarantino A, Santoro N, et al. Wernicke's encephalopathy induced by total parenteral nutrition in patient with acute leukaemia: unusual involvement of caudate nuclei and cerebral cortex on MRI. Neuroradiology 2000;42:781-83

18. Peters TE, Parvin M, Petersen C, et al. A case report of Wernicke's encephalopathy in a pediatric patient with anorexia nervosa-restricting type. J Adolesc Health 2007;40:376-83

19. Butterworth RF, Gaudreau C, Vincelette J, et al. Thiamine deficiency and Wernicke's encephalopathy in AIDS. Metab Brain Dis 1991;6:207-12

20. Fattal-Valevski A, Kesler A, Sela BA, et al. Outbreak of life-threatening thiamine deficiency in infants in Israel caused by a defective soy-based formula. Pediatrics 2005; 115:e233-38

21. Attard O, Dietemann JL, Diemunsch P, et al. Wernicke encephalopathy: a complication of parenteral nutrition diagnosed by magnetic resonance imaging. Anesthesiology 2006;105:847-48

22. Francini-Pesenti F, Brocadello F, Famengo S, et al. Wernicke's encephalopathy during parenteral nutrition. JPEN J Parenter Enteral Nutr 2007;31:69-71

23. Basoğlu M, Yetimalar Y, Gürgör N, et al. Neurological complications of prolonged hunger strike. Eur J Neurol 2006;13:1089-97

24. Drenick EJ, Joven CB, Swendseid ME. Occurrence of acute Wernicke's encephalopathy during prolonged starvation for the treatment of obesity. $N$ Engl J Med 1966;274:937-39

25. Yae S, Okuno S, Onishi H, et al. Development of Wernicke encephalopathy in a terminally ill cancer patient consuming an adequate diet: a case report and review of the literature. Palliat Support Care 2005;3:333-35

26. Ihara M, Ito T, Yanagihara C, et al. Wernicke's encephalopathy associated with hemodialysis: report of two cases and review of the literature. Clin Neurol Neurosurg 1999;101:118-2

27. Baek JH, Sohn SK, Kim DH, et al. Wernicke's encephalopathy after allogeneic stem cell transplantation. Bone Marrow Transplant 2005;35:829-30
28. Thaisetthawatkul P, Collazo-Clavell ML, Sarr MG, et al. A controlled study of peripheral neuropathy after bariatric surgery. Neurology 2004;63:1462-70

29. Sechi G, Serra A. Wernicke's encephalopathy: new clinical settings and recent advances in diagnosis and management. Lancet Neurol 2007;6:442-55

30. Liu YT, Fuh JL, Lirng JF, et al. Correlation of magnetic resonance images with neuropathology in acute Wernicke's encephalopathy. Clin Neurol Neurosurg 2006;108:682-87

31. Vasconcelos MM, Silva KP, Vidal G, et al. Early diagnosis of pediatric Wernicke's encephalopathy. Pediatr Neurol 1999;20:289-94

32. Reuler JB, Girard DE, Cooney TG. Current concepts. Wernicke's encephalop athy. $N$ Engl J Med 1985;312:1035-39

33. Nordentoft M, Timm S, Hasselbalch E, et al. Thiamine pyrophosphate effect and erythrocyte transketolase activity during severe alcohol withdrawal syndrome. Acta Psychiatr Scand 1993;88:80-84

34. Antunez E, Estruch R, Cardenal C, et al. Usefulness of CT and MR imaging in the diagnosis of acute Wernicke's encephalopathy. AJR Am J Roentgenol 1998;171:1131-37

35. Gallucci M, Bozzao A, Splendiani A, et al. Wernicke encephalopathy: MR findings in five patients. AJNR Am J Neuroradiol 1990;11:887-92

36. Chung SP, Kim SW, Yoo IS, et al. Magnetic resonance imaging as a diagnostic adjunct to Wernicke encephalopathy in the ED. Am J Emerg Med 2003;21:497-502

37. Weidauer S, Nichtweiss M, Lanfermann H, et al. Wernicke encephalopathy: MR findings and clinical presentation. Eur Radiol 2003;13:1001-09

38. Park SH, Kim M, Na DL, et al. Magnetic resonance reflects the pathological evolution of Wernicke encephalopathy. J Neuroimaging 2001;11:406-11

39. Shogry ME, Curnes JT. Mamillary body enhancement on MR as the only sign of acute Wernicke encephalopathy. AJNR Am J Neuroradiol 1994;15:172-74

40. D'Aprile P, Gentile MA, Carella A. Enhanced MR in the acute phase of Wernicke encephalopathy. AJNR Am J Neuroradiol 1994;15:591-93

41. Charness ME. Brain lesions in alcoholics. Alcohol Clin Exp Res 1993;17:2-11

42. Nicolás JM, Fernández-Solà J, Robert J, et al. High ethanol intake and malnutrition in alcoholic cerebellar shrinkage. QJM 2000;93:449-56

43. Halavaara J, Brander A, Lyytinen J, et al. Wernicke's encephalopathy: is diffusion-weighted MRI useful? Neuroradiology 2003;45:519-23

44. Bae SJ, Lee HK, Lee JH, et al. Wernicke's encephalopathy: atypical manifestation at MR imaging. AJNR Am J Neuroradiol 2001;22:1480 - 82

45. Opdenakker G, Gelin G, De Surgeloose D, et al. Wernicke encephalopathy: MR findings in two patients. Eur Radiol 1999;9:1620-24

46. Doss A, Mahad D, Romanowski CA. Wernicke encephalopathy: unusual findings in nonalcoholic patients. J Comput Assist Tomogr 2003;27:235-40

47. Kumral E, Evyapan D, Balkir K, et al. Bilateral thalamic infarction. Clinical, etiological and MRI correlates. Acta Neurol Scand 2001;103:35-42

48. Schmahmann JD. Vascular syndromes of the thalamus. Stroke 2003;34 2264-78

49. Forsting M, Krieger D, Seier U, et al. Reversible bilateral thalamic lesions caused by primary internal cerebral vein thrombosis: a case report. J Neurol 1989;236:484-86

50. Pilleri G, Pietrini V, Tagliavini F, et al. "Thalamic" dementia in herpes encephalitis: clinico-pathological report. Acta Neuropathol Suppl (Berl) 1981;7:156-59

51. Gerwig M, Kastrup O, Wanke I, et al. Adult post-infectious thalamic encephalitis: acute onset and benign course. Eur J Neurol 2004;11:135-39

52. Carota A, Pizzolato GP, Gailloud P, et al. A panencephalopathic type of Creutzfeldt-Jakob disease with selective lesions of the thalamic nuclei in 2 Swiss patients. Clin Neuropathol 1996;15:125-34

53. Matheus MG, Castillo M. Imaging of acute bilateral paramedian thalamic and mesencephalic infarcts. AJNR Am J Neuroradiol 2003;24:2005-08

54. Miyakawa E, Tokumaru AM, O'uchi T, et al. A case of bilateral reversible thalamic lesions. Eur Radiol 1997:7:470-73 